\title{
Perampanel overdose in low body mass index patients with epilepsy: a case report and review of the literature
}

\author{
Kanitpong Phabphal ${ }^{*}[$ and Prut Koonalintip
}

\begin{abstract}
Background: Perampanel (PER) is a novel antiepileptic drug (AED) which employs a completely different mechanism of action compared to existing medications. Overall, PER is considered to be safe up to a dose of $12 \mathrm{mg}$ per day. When used to treat refractory and super-refractory status epilepticus, PER seems to be extremely well tolerated; this is true even when used at doses of up to $32 \mathrm{mg}$. There are currently only three case reports on the effects of acute PER overdose in epilepsy patients.

Case presentation: We report a 16-year-old Thai woman with a low body weight, who took PER at a dose of 40 times that of the prescribed daily dose. She experienced only an alteration of consciousness, without any systemic medical effects, and made a full recovery within 3 days without gastric lavage or specific treatment.

Conclusion: Our report demonstrates that an acute PER overdose may not produce serious adverse systemic effects. Individuals with adverse central nervous system (CNS) effects, such as altered consciousness, can experience a rapid recovery.
\end{abstract}

Keywords: Perampanel, Overdose, Epilepsy

\section{Background}

Among the currently available antiepileptic drugs (AEDs), perampanel (PER) is a novel AED with a completely different mechanism of action. It is the first selective, noncompetitive $\alpha$-amino-3-hydroxy-5-methyl4-isoxazolepropionic acid (AMPA) receptor antagonist used to treat epilepsy. Clinical trials have shown that PER is generally well tolerated, though dose-dependent central nervous system (CNS) effects occur. In the short term, the most commonly reported treatment-emergent adverse events (TEAEs) leading to dose reductions or treatment interruptions are dizziness, somnolence, headache, and fatigue [1]. PER, at $2 \mathrm{mg} / \mathrm{day}$, has been demonstrated to have similar effects and side effects as those

*Correspondence: pkanitpo@gmail.com

Neurology Unit, Department of Internal Medicine, Faculty of Medicine,

Prince of Songkla University, Hat Yai, Songkhla 90110, Thailand of a placebo [2]. At doses of $4 \mathrm{mg} /$ day, dizziness, somnolence, and irritability have been reported as adverse events (AEs). TEAEs are generally more often reported at doses of 8 or $12 \mathrm{mg} /$ day [3]. An extension study of a trial held over a period of 4 years revealed that the most common TEAEs leading to PER therapy discontinuation were dizziness (4.1\%), irritability (1.2\%), and fatigue (1.1\%) [4]. The efficacy of perampanel has been evaluated extensively in various phase II, randomized placebo-controlled phase III [1,2], and open-label extension trials [5]. The patients recruited in these studies were $\geq 12$ years of age, had refractory partial seizures with or without secondary generalization, and were on 1-3 additional AEDs $[2,4,6]$.

The usual starting dose of PER for focal and generalized seizures in adult patients who are not concomitantly taking an enzyme-inducing antiepileptic drug is $2 \mathrm{mg} /$ day before sleep. Additionally, it is recommended that the dose be increased by $2 \mathrm{mg} /$ day biweekly to reach the original author(s) and the source, provide a link to the Creative Commons licence, and indicate if changes were made. The images or other third party material in this article are included in the article's Creative Commons licence, unless indicated otherwise in a credit line to the material. If material is not included in the article's Creative Commons licence and your intended use is not permitted by statutory regulation or exceeds the permitted use, you will need to obtain permission directly from the copyright holder. To view a copy of this licence, visit http://creativecommons.org/licenses/by/4.0/. The Creative Commons Public Domain Dedication waiver (http://creativeco mmons.org/publicdomain/zero/1.0/) applies to the data made available in this article, unless otherwise stated in a credit line to the data. 
maximum tolerable dose (usually $4-8 \mathrm{mg} /$ day), which provides excellent seizure control. Overall, PER is considered to be safe up to a daily dose of $12 \mathrm{mg}$ per day. When used to treat refractory and super-refractory status epilepticus (SE), PER seems to be extremely well tolerated; the same holds true even when used at doses of up to 32 mg. However, currently, very little has been published about incidents where PER is taken above the recommended dosages [7-10]. Three patients with epilepsy [7-9] and one patient with depression and anxiety [10] have been reported to have experienced PER intoxication. Those reports included adult patients aged 34, 39, 40 , and 54 years. None of the four studies on PER intoxication reported the body mass index (BMI) of the participants. However, all participants enrolled in the core study investigating the efficacy of PER had a normal BMI. With regard to the pharmacokinetics, PER undergoes extensive hepatic metabolism $(>90 \%)$ to form 13 major metabolites of hydroxylated perampanel and various glucuronide conjugates. The isoenzyme CYP3A4 is considered to be primarily involved in the metabolism of PER [11]. In a recent study on the correlation between body weight and CYP3A activity, [12] Sandvik et al. reported that the enzyme activity of CYP3A4 was higher in patients with anorexia nervosa compared to a control group consisting of individuals with normal weight, and that CYP3A4 activity was negatively correlated with body weight [13]. Also, CYP3A4 activity was found to decrease with increasing body weight in healthy volunteers [14].

Herein, we describe the clinical findings in a low-BMI patient with epilepsy, who ingested an estimated $80 \mathrm{mg}$ of PER in a suicide attempt. This study was approved by the institutional review board of the Faculty of Medicine, Prince of Songkla University (REC.62-396-14-3).

\section{Case presentation}

The patient was a 16-year-old Thai female who had suffered from refractory epilepsy since the age of 10 . She had a focal onset with impaired awareness and motor predominance at least five times per month. Magnetic resonance imaging studies revealed a non-enhancing diffuse enlargement of the right amygdala, hippocampus, and parahippocampal gyrus, and electroencephalography showed occasional spikes in the corresponding areas. She weighed $32 \mathrm{~kg}(\mathrm{BMI}=19)$. She had been on multiple antiepileptic drugs, which include valproate $2000 \mathrm{mg} /$ day, levetiracetam $1500 \mathrm{mg} /$ day, and lamotrigine $50 \mathrm{mg} /$ day. At the age of 16 , she experienced multiple attacks of convulsive SE, so she was transferred to our hospital for more specialized treatment. A mood disorder was detected on that visit, but no relevant mood conditions before or during treatment at our department were reported by either the patient or her family.
Moreover, neither suicidal thoughts nor previous suicide attempts were reported. The patient was initially treated by increasing the lamotrigine dose to $100 \mathrm{mg} /$ day, but her seizures persisted. Two milligrams per day of PER before sleep was added as adjunctive therapy; the dose was uptitrated to $4 \mathrm{mg} /$ day after 2 weeks. Subsequently, she was seizure-free for 6 months. The seizures were controlled with combination therapy consisting of $4 \mathrm{mg} /$ day of PER, the withdrawal of valproate and levetiracetam, and the tapering of lamotrigine to $25 \mathrm{mg} /$ day. We then decided to switch to PER monotherapy by tapering off lamotrigine. The patient had been seizure-free for 8 months when she was found drowsy in her bedroom by her friends, with an empty bottle of PER; there were approximately 40 tablets missing $(80 \mathrm{mg})$. On physical examination, her mental status was described as a state of confusion, and she exhibited signs of withdrawal in response to painful stimuli. Her Glasgow Coma Scale (GCS) score was 8. The other physical findings were as follows: normal vital signs; skin that felt warm to the touch; and pupils that were equal, round, and reactive to light. In addition, her reflexes were normal, there were no focal neurological signs or symptoms, and her mucous membranes were moist. Moreover, no tremors were noted, and her gag reflex was intact. No specific treatment was administered in the emergency room; specifically, no gastric lavage was performed because the time of ingestion was more than 3 hours prior to presentation. It is known that oral PER is rapidly and almost completely absorbed (time to maximum concentration $\left[T_{\max }\right], 0.5-2.5$ hours), is not subject to any significant first-pass metabolism, and has a bioavailability of almost $100 \%$. Her initial laboratory results were normal, and comprised the findings of a complete blood count, electrolyte test, liver function test, and renal function test. A urine drug and toxic substance screen was negative for benzodiazepines, amphetamine, and ethanol. Additional testing including head computed tomography $(\mathrm{CT})$, chest X-ray, and electrocardiography showed no acute abnormalities. An electroencephalogram performed on the day after admission demonstrated a diffuse slowing and disorganization of the background, consistent with generalized dysfunction. Unfortunately, we were unable to determine serum concentrations due to the unavailability of the technology to measure the blood levels of PER in Thailand.

During the patient's hospital stay, her symptom of drowsiness changed to a qualitative impairment of consciousness involving disorientation and misjudgment of situations, which resolved in 2 days (full GCS score). Her vital signs and other laboratory results remained stable during the first 2 days of admission, and there was no evidence of perampanel-induced systemic AEs such as hepatic or renal toxicity, hypotension, or respiratory 
suppression. Throughout hospitalization, her vital signs remained within normal parameters. She was discharged on admission day 3 with a normal level of consciousness as well as other neurological and general medical parameters. Once medically cleared, she voluntarily agreed to transfer to the psychiatry ward. During her time there, she was diagnosed with severe depression induced by familial problems. During consultation with the attending staff there, she said, "It was just a blind rage; I did not mean to do that. I am so sorry". The psychiatric condition leading to her suicide attempt was the diagnosed major depressive syndrome. She was put on an antidepressant, and her depressive symptoms partially resolved. She was deemed psychiatrically stable and was discharged with a prescription of sertraline $50 \mathrm{mg}$ daily and outpatient psychiatric follow-up care. At the initial follow-up 2 weeks after discharge, she reported mood changes with depressive symptoms, which had begun after the tapering off of lamotrigine, and at the same time she was experiencing some family problems; she did not notify her doctor about this. Two months after discharge, she was taking lamotrigine $100 \mathrm{mg} /$ day and sertraline $50 \mathrm{mg} /$ day.

\section{Discussion}

There is sparse information regarding the effects of PER in cases of an acute overdose. Hence, the definition of PER overdose is not clear. However, we defined it as having received more than $32 \mathrm{mg} /$ day of PER. Herein, we report on a patient from Thailand with a low BMI who overdosed on PER $80 \mathrm{mg} /$ day. Our patient was prescribed a relatively low dose ( $4 \mathrm{mg} /$ day), so the dose she ingested $(80 \mathrm{mg} /$ day) was 20 times higher than the upper limit of her daily dose. The neurological signs in this case involved a significantly depressed mental status manifested by her symptom of drowsiness. Interestingly, her symptoms persisted for 2 days, after which a rapid and complete recovery of her clinical condition was observed. These findings are in contrast to those reported by Kim et al. involving a 39-year-old woman admitted due to altered consciousness and stupor after taking 10 times the daily dose of both PER and valproic acid (she had been taking $4 \mathrm{mg} /$ day of PER and $2000 \mathrm{mg}$ of valproate). In that case, the patient began to regain consciousness on hospitalization day 6 and returned to her baseline mental status on day 8 [7]. Likewise, Li et al. reported prolonged stupor in a 54-year-old man who had experienced PER intoxication [8].

Our patient ingested 20 times the prescribed PER dosage, which is double that reported by Kim et al. The reason why our patient recovered so quickly from altered consciousness may be the fact that she took only PER without any concomitant drugs, especially enzyme inhibitors, whereas the case reported by Kim et al. had taken
PER and valproic acid (an enzyme inhibitor antiepileptic drug). There are at least three routes of valproate metabolism: glucuronidation (50\%), $\beta$ oxidation (40\%) in the mitochondria, and cytochrome P450 (CYP)-mediated oxidation (10\%) [15] - then did not effect PER metabolism. Kim et al. believed that the prolonged stupor in that case was due to the prolonged half-life of PER, not the result of valproate encephalopathy. Another possible explanation is the low BMI of our patient. A previous study reported a negative correlation between BMI and CYP3A4 levels. CYP3A4 is a major metabolite of PER, and an increase in the level of CYP3A4 results in an increase in PER metabolism. Nevertheless, a comprehensive understanding of this mechanism is lacking and requires further study. According to clinical trials, the most commonly reported AEs from therapeutic dosing are dizziness, somnolence, fatigue, and irritability $[3,4]$. However, little information on the effects of PER overdose is available. Case reports on PER overdose have revealed that the most frequent clinical presentations involve altered consciousness (stupor) and severe aggression (Table 1) [7-10].

With respect to the systemic AEs of PER, data related to vital signs, clinical laboratory parameters, and electrocardiographic findings $[1,2,16]$ have revealed that patients taking PER in combination therapy are more likely to experience AEs than those on PER monotherapy. Additionally, a dose-response relationship was discovered. Overall, most reported TEAEs were considered mild or moderate in severity; no deaths were recorded, but severe TEAEs were reported by 22 patients $(5.0 \%)$ receiving placebo and 57 (5.5\%) receiving PER at any dose. The serious TEAEs included falls, nephrolithiasis, cholelithiasis, hemorrhagic cystitis, urinary incontinence, and thrombocytopenia. There have been no reports of unstable vital signs, liver toxicity, abnormal complete blood count findings, or cardiac conduction defects. In terms of PER overdose cases, Wu et al. reported a psychiatric patient with a PER overdose and a urine screen that was positive for benzodiazepine and ethanol. They described a patient with severe respiratory depression, who was unresponsive. He was intubated due to concerns related to his airway protection [10]. Another report by Li et al. involving epileptic patients with PER overdose described findings of asymptomatic bradycardia and hyponatremia with spontaneous recovery without specific treatment [8]. Our patient reportedly ingested up to $80 \mathrm{mg}$ of PER (40 times her daily dose) and experienced a short period of drowsiness with a significantly depressed mental status and minimal reaction to painful stimuli while maintaining her airway reflexes, which did not warrant endotracheal intubation. In addition, our report points out that an acute PER overdose may not produce serious adverse systemic 


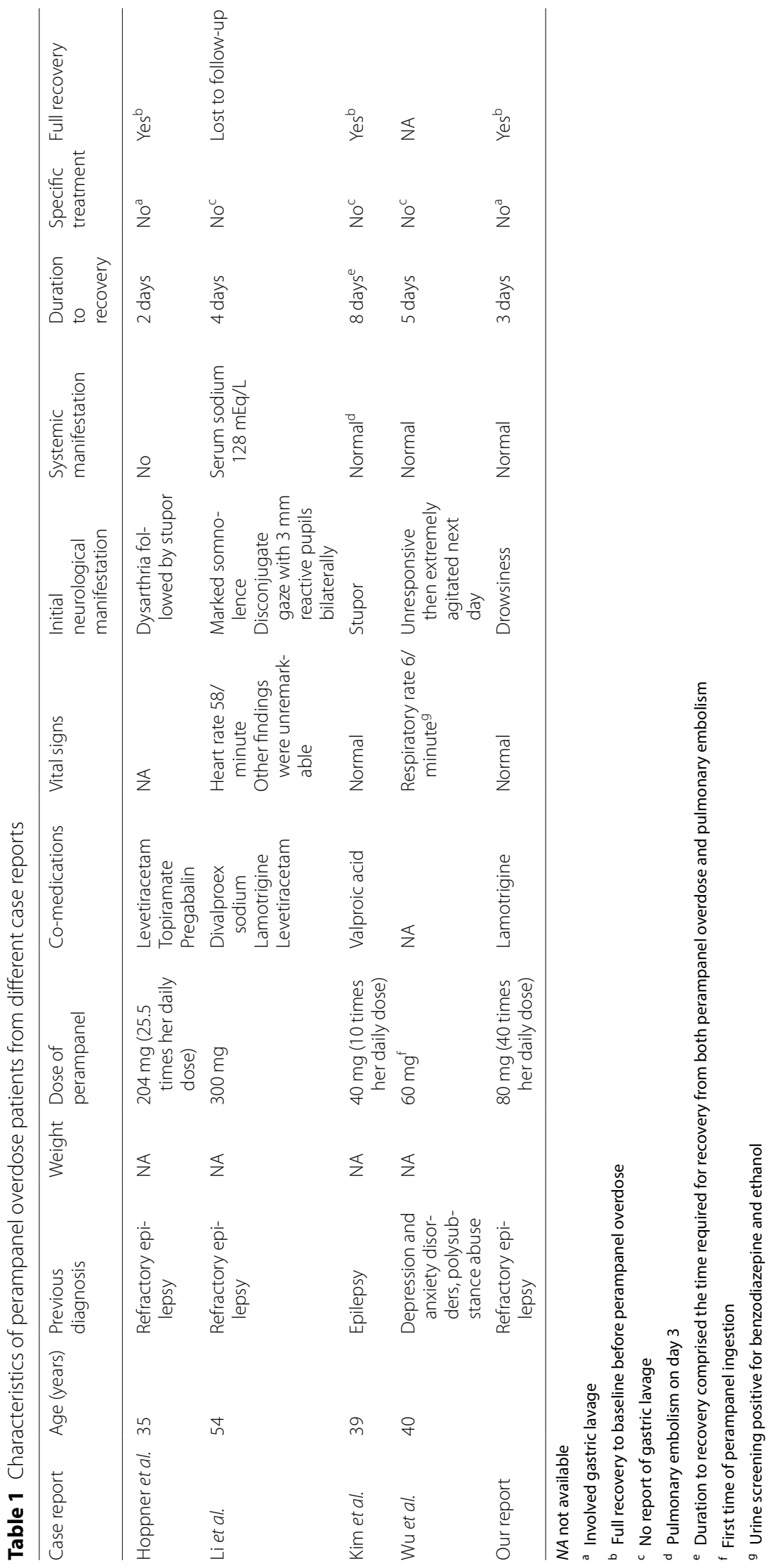


effects such as cardiac toxicity, respiratory depression, or other metabolic derangement symptoms. This finding is supported by the results reported by Hopper et al. [9] and Kim et al. [7], which involved patients that had intentionally overdosed on PER as a suicide attempt and experienced symptoms such as impaired consciousness and agitation, without any significant systemic AEs.

As of now, there is no specific treatment for PER overdose. With regard to its pharmacokinetic properties, the PER concentration has been found to peak after $60 \mathrm{~min}$ utes. In phase I studies among healthy male volunteers, the mean plasma half-life of PER was found to range from 52 to 129 hours following a single-dose administration; this could explain our patient's short duration of coma. If patients present to the hospital more than 60 minutes after ingestion, gastric lavage may not be necessary. According to previous single-case reports, patients recover 2 to 5 days after admission [7-10] without gastric lavage or specific treatment. However, the plasma half-life of PER may be shorter or longer than that of a single dose of PER in the presence of co-medication with cytochrome P450 enzyme inducers or inhibitors.

\section{Conclusion}

Our report demonstrates that an acute PER overdose may not produce serious adverse systemic effects such as cardiac toxicity, respiratory depression, or other metabolic derangement symptoms. In addition, adverse CNS side effects such as altered consciousness may experience rapid recovery.

\section{Abbreviations}

AEDs: Antiepileptic drugs; AMPA: a-Amino-3-hydroxy-5-methyl-4isoxazolepropionic acid; PER: Perampanel; SE: Status epilepticus.

\section{Acknowledgements}

We would like to thank Mr. Edmond Subashi for editing the English manuscript.

\section{Authors' contributions}

All authors contributed to the care of this patient. All authors contributed to the original draft and revisions of the manuscript. All authors read and approved the final manuscript.

\section{Funding}

No funding.

\section{Availability of data and materials \\ Not applicable.}

\section{Ethics approval and consent to participate}

Institutional review board approval was obtained from the Ethics Committee, Faculty of Medicine, Prince of Songkla University

\section{Consent for publication}

Written informed consent was obtained from the patient's legal guardian(s) for the publication of this case report and any accompanying images. A copy of the written consent is available for review by the Editor-in-Chief of this journal upon request.

\section{Competing interests}

The authors declare that they have no competing interests.

Received: 28 September 2020 Accepted: 23 February 2021

Published online: 29 March 2021

\section{References}

1. French J, Krauss G, Biton $\mathrm{V}$, et al. Adjunctive perampanel for refractory partial-onset seizures: randomized phase III study 304. Neurology. 2012:79:589-96.

2. Krauss GL, Serratosa JM, Villanueva V, et al. Randomized phase III study 306: adjunctive perampanel for refractory partial-onset seizures. Neurology. 2012;78:1408-15.

3. Serratosa JM, Villanueva V, Kerling F, Kasper BS. Safety and tolerability of perampanel: a review of clinical trial data. Acta Neurol Scand Suppl. 2013;197:30-5.

4. Krauss G, Perucca E, Kwan P, et al. Final safety, tolerability, and seizure outcomes in patients with focal epilepsy treated with adjunctive perampanel for up to 4 years in an open-label extension of the phase III randomized trials: study 307. Epilepsia. 2018;59:866-76

5. Montouris G, Yang H, Williams B, Zhou S, Laurenza A, Fain R. Efficacy and safety of perampanel in patients with drug-resistant partial seizures after conversion from double-blind placebo to open-label perampanel. Epilepsy Res. 2015a;114:131-40.

6. Montouris G, Yang H, Williams B, Zhou S, Laurenza A, Fain R. Efficacy and safety of perampanel in patients with drug-resistant partial seizure after conversion from double-blind placebo to open-label perampanel. Epilepsy Res. 2015b;114:131-40.

7. Kim S, Kim TE, Kim D, Kim DW. Prolonged stupor in perampanel overdose and pharmacokinetic considerations. J Epilepsy Res. 2018:8:87-9.

8. Li K, Lasoff DR, Smollin CG, Ly BT. Perampanel overdose causing a prolonged coma. Clin Toxicol (Phila). 2018;56:677-8.

9. Hoppner AC, Falser S, Kerling F. Clinical course of intoxication with the new anticonvulsant drug perampanel. Epileptic Disord. 2013;15:362-4.

10. Wu CC, MCShane M, Huttlin EA, Novoa KC. Severe aggression after perampanel overdose: case report. Psychosomatics. 2019;60:321-4.

11. Patsalos PN. The clinical pharmacology profile of the new antiepileptic drug perampanel: a novel noncompetitive AMPA receptor antagonist. Epilepsia. 2014:56:12-27.

12. Krogstad V, Peric A, Robertsen I. Correlation of body weight and composition with hepatic activities of cytochrome P450 enzymes. J Pharm Sci. 2021;110:432-7.

13 Sandvik P, Lydersen S, Hegstad S, Spigset O. Association between low body weight and cytochrome P-450 enzyme activity in patients with anorexia nervosa. Pharmacol Res Perspect. 2020;8:00615.

14. Ulvestad M, Skotheim IB, Jakobsen GS, et al. Impact of OATP1B1, MDR1, and CYP3A4 expression in liver and intestine on interpatient pharmacokinetic variability of atorvastatin in obese subjects. Clin Pharmacol Ther. 2013;93:275-82.

15 Davis R, Peters DH, McTavish D. Valproic acid. A reappraisal of its pharmacological properties and clinical efficacy in epilepsy. Drugs. 1994;47:332-72.

16 French JA, Krauss GL, Steinhoff BJ, et al. Evaluation of adjunctive perampanel in patients with refractory partial-onset seizures: results of randomized global phase III study 305. Epilepsia. 2013;54:117-25.

\section{Publisher's Note}

Springer Nature remains neutral with regard to jurisdictional claims in published maps and institutional affiliations. 